\title{
Percutaneous mechanical thrombectomy for treatment of acute femoropopliteal bypass occlusion
}

This article was published in the following Dove Press journal:

Vascular Health and Risk Management

2 May 2012

Number of times this article has been viewed

\author{
Michael Lichtenberg' \\ Matthias Käunicke' \\ Birgit Hailer ${ }^{1,2}$ \\ 'Cardiovascular Clinic, Vascular \\ Center, Katholisches Klinikum Essen, \\ Germany; ${ }^{2}$ University of Witten/ \\ Herdecke, Germany
}

\begin{abstract}
Acute and subacute ischemia of the legs in acute and subacute femoropopliteal bypass occlusion is a dramatic situation that endangers the survival of the limbs, depending on the severity of the ischemia. Different therapy options like percutaneous mechanical thrombectomy procedures, which include rotational thrombectomy, have become available in recent years, in addition to local lysis and surgical thrombectomy. Surgical thrombectomy using the Fogarty catheter technique, in particular, shows an increased incidence of perioperative complications but only small technical success rates in randomized trials. On the other hand, local lysis is associated with increased costs due to resource-consuming measures, such as intensive monitoring and repeat angiographies, in addition to bleeding complications. In the past, further development of the Straub Rotarex ${ }^{\circledR}$ system as an endovascular therapy option has demonstrated good success leading to amputation-free survival in multiple studies. At the same time, a low rate of complications with use has been documented. Most examinations have been conducted in the thigh. To date, there are little investigational data on its use in acutely and subacutely occluded femoropopliteal bypasses. In this paper, the current study-based significance of the Rotarex system for this indication is analyzed based on the existing literature and the authors' own experiences with 22 patients.
\end{abstract}

Keywords: acute limb ischemia, femoropopliteal bypass, local lysis, rotational thrombectomy

\section{Introduction}

Acute ischemia of the limbs is characterized by a significant reduction in arterial perfusion, which, in addition to the danger of injury to the limbs including amputation, can mean life-threatening complications for affected patients. The existing hypoperfusionrelated anaerobic local as well as systemic metabolic situation can have direct negative effects on other organ systems such as the kidneys, brain, and heart. The therapeutic management to be immediately established therefore includes the decision regarding appropriate reperfusion measures in addition to general, mostly intensive care, measures. In the case of acute femoropopliteal bypass occlusions, local lysis has also been established as an alternative measure in addition to the established vascular intervention (Fogarty maneuvers, endarterectomy - another bypass operation). Therapeutic recommendations based on large randomized studies on local lysis can be found on this in the current TASC Working Group Guidelines. ${ }^{1-4}$ Percutaneous mechanical thrombectomy (PMT) has also been proven as an additional therapy option in recent years. Because of good study data from the interventional therapy of acute and subacute arterial occlusion, more and more attention has been paid to rotational thrombectomy. ${ }^{5}$ In the meantime, the Rotarex $^{\circledR}$ system (Straub Medical, Wangs, Switzerland), with technical enhancements,
Correspondence: Michael Lichtenberg Cardiovascular Clinic, Vascular Center, Katholisches Klinikum Essen,

Huelsmannstraße I7, D-45355

Essen, Germany

Tel +492016400330 I

Fax +49 20I 64003304

Email m.lichtenberg@kk-essen.de

\footnotetext{
Vascular Health and Risk Management 201 2:8 283-289 
has become a relevant thrombectomy treatment for arterial vessel occlusions. Zeller and Wissgott et al in particular have described favorable courses with the Rotarex in systematic study analyses following acute recanalization. ${ }^{6-8}$

The objective of the prospective study was to analyze the efficacy of the device in the indication of acute femoropopliteal bypass occlusions in 22 patients. The patients were followed over a period of 6 months for clinical outcome and patency of the femoropopliteal bypass.

\section{PMT with the Rotarex system}

The Straub Rotarex thrombectomy system works on the Archimedean screw principle, which is generated by a spiral rotating at a speed of approximately 40,000 revolutions per minute.

The system consists of three individual components that can be put together by an experienced team within a few minutes. It is operated by a motor unit with an electronic control unit, which also displays information on the functionality of the rotating helix. Inside the catheter runs a helical conveyor drive, which is connected to the motor unit via a magnetic coupling. The rapid rotation of the helix generates a permanent vacuum inside the catheter, which aspirates the thrombotic material in the target lesion and conveys it into a collection bag at the end of the catheter. Depending on the size of the system used (Rotarex 6-8 F, Aspirex ${ }^{\circledR}$ 6-10 F), aspiration capacities of up to $1.5 \mathrm{~mL} / \mathrm{s}$ can be achieved for the $8 \mathrm{~F}$ Rotarex system. The larger systems are especially used in the area of the pelvis and thigh where thrombotic occlusions mean larger vessel diameters to deal with and greater volumes of thrombotic material to be removed. Depending on the size of the target vessel, the 6 F system can be used to conduct a thrombectomy far into the lower leg. A standard $4 \mathrm{~F}$ system for distal lower leg procedures is in the planning stages, according to the manufacturer, and could provide therapy options up into the foot region.

After conducting a diagnostic angiography, a $6 \mathrm{~F}$ or $8 \mathrm{~F}$ diameter Rotarex system should be used, depending on the vessel diameter. Then, using roadmap or overlay technique, a 0.018 inch guide wire is inserted up to the target lesion and advanced distally beyond it. The Rotarex system is advanced over this guide wire up to a few centimeters above the thrombotic occlusion and then activated. The occlusion should be passed slowly. A slow passage is especially recommended for subacute occlusions with material that is already partially organized in order to prevent peripheral embolisms (see Figure 2).

Small forward and backward movements should be made. Depending on the final morphology after reestablishing flow, an angioplasty with or without stent implantation must be considered, in keeping with the current recommendations. We conducted effective antithrombotic therapy with heparin during the procedure, with managed ACT (200-250 seconds). Saturation with acetylsalicylic acid was also carried out. If a stent was implanted, clopidogrel saturation with $600 \mathrm{mg}$ was performed, with subsequent continuation for 4 weeks.

\section{Methods and results}

From June 2008 to June 2010, a total of 22 patients (twelve male) with acute occlusions of a femoropopliteal bypass (twelve venous bypasses, ten polytetrafluoroethylene [PTFE] bypasses) were treated with the Rotarex system in our clinic (Tables 1 and 2, Figures 3-6). In this tracked study group, only P1 bypasses (proximal popliteal artery) were treated and evaluated. Clinically, ten patients exhibited stage I and twelve patients stage IIa acute ischemia of the extremities according to the recognized TASC classification. The presumed duration of the occlusions, according to the patients' medical histories (onset of complaints to clinical presentation) was $10 \pm 6$ days. The average ankle-brachial index (ABI) value before the procedure was $0.42 \pm 0.1$ on the affected side. The age of the bypasses could not be determined for certain due to divergent statements; therefore, a decision was made not to indicate the average age of the bypasses. In ten patients, a high-grade to filiform bypass insertion stenosis was shown angiographically as a possible cause of the femoropopliteal bypass occlusion, which was treated in each case with a nitinol stent implantation (nine patients) or a conventional balloon angioplasty (one patient). None of the patients had an upstream bypass stenosis. In six patients, the presumed cause was determined to be a cardioembolic event in subsequent examinations. One patient had a large aneurysm of an approximately 18-year-old venous bypass upstream of the distal anastomosis. Here, the

\section{Table I Patient data}

\begin{tabular}{ll}
\hline Patients (n) & 22 \\
Age (years) & $70.2 \pm 15.3$ \\
Men & 12 \\
Women & 10 \\
Smokers & 15 \\
Ex-smokers & 5 \\
Dyslipoproteinemia & 18 \\
Diabetes mellitus & 10 \\
Hypertension & 16 \\
Coronary artery disease (CAD) & 12 \\
ABI value prior to procedure & 0.41 \\
Stage I ischemia & 10 \\
Stage Ila ischemia & 12 \\
\hline
\end{tabular}


Table 2 Vessel data

Length of occlusion in femoropopliteal bypass $(\mathrm{cm})$

$28 \pm 10 \mathrm{~cm}$

Venous bypass

12

Artificial bypass graft (PTFE)

PI segment femoropopliteal bypass

Age of bypass (months)

Crural outflow vessels

10

22
2-120 months

3 ( 10 patients)

2 (8 patients)

I (4 patients)

Abbreviation: PTFE, polytetrafluoroethylene.

aneurysm was eliminated with a Viabahn ${ }^{\circledR}$ endoprosthesis in a second procedure since the patient refused a revision operation (Figure 7). The thrombectomy was conducted in twelve patients using the $8 \mathrm{~F}$ Rotarex system, and in the remaining patients with the $6 \mathrm{~F}$ system. All procedures were conducted using crossover technique; for this reason, the $110 \mathrm{~cm}$ Rotarex shaft length was used in all cases. The crossover maneuver was possible in all cases. Prior to the procedure, the patients received a heparin bolus of $5000 \mathrm{IU}$ heparin and $500 \mathrm{mg}$ aspirin. In the cases in which a nitinol stent had to be implanted, saturation with clopidogrel $600 \mathrm{mg}$ was performed while the patient was still in the angiography lab, and then clopidogrel $75 \mathrm{mg}$ was recommended for these patients for 4 weeks. Overall, an average of $4 \pm 2$ thrombectomy passes were performed with the Rotarex system, beginning each time proximally in the occlusion with light forward and backward movements. The supplied 0.018 inch guide wires were used as the standard guide wire in almost all cases. In all cases, this guide wire was able be guided through the thrombotic occlusion. To verify a safe intravascular position of the guide wire, a local injection of contrast agent was performed in all patients via an over-the-wire catheter, which was inserted beforehand over the guide wire into the periphery. The average occlusion length was about $30 \mathrm{~cm}$. In five patients, due to the detection of a thrombus in popliteal artery segments II and III, a rotation thrombectomy also had to be performed in these vessel segments,

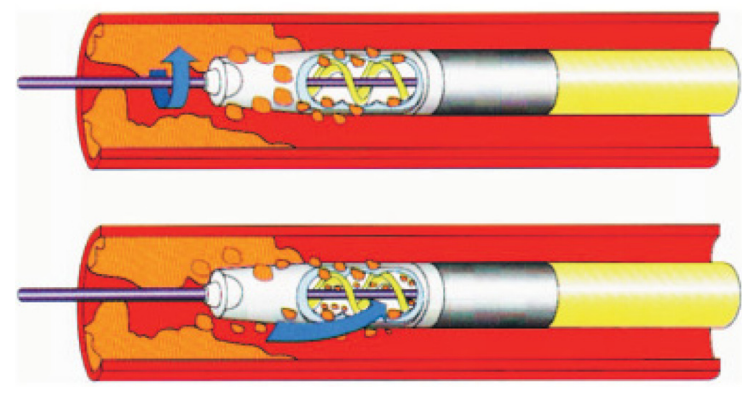

Figure I The Rotarex ${ }^{\circledR}$ system.

\section{Contraindications}

- If it is impossible to penetrate the lesion with the guide wire

- Subintimal position

- Use in vessels with too small diameter (<4 mm)

- If it is impossible to achieve sufficient anticoagulation

- Patient with coagulation system disorders

Figure 2 Contraindications for Rotarex ${ }^{\circledast}$ use.

each time with a good early angiographic result. None of the patients experienced a vessel dissection or perforation. In four patients, local lysis still had to be performed after the Rotarex thrombectomy via an appropriate catheter since significant residual thrombi were still detected after several thrombectomy passes. Because of the persisting thrombus load, local lysis was performed in this patient with a tissueplasminogen activator (Actilyse ${ }^{\circledR}$ ) for up to 24 hours (bolus $5 \mathrm{mg}, 1-3 \mathrm{mg} / \mathrm{h}$ ) with a pulse-spray catheter. The average ABI value after the procedure was $0.85 \pm 0.1$. Six months after the index procedure follow-up studies were performed on 21 out of 22 patients. Follow up included ABI measurement, clinical examination of the patient, and a Doppler and duplex study of the femoropopliteal bypass and the outflow vessels. The walking capacity was measured using a standardized treadmill test in the patients who were able to perform this test. One patient refused follow-up examination.

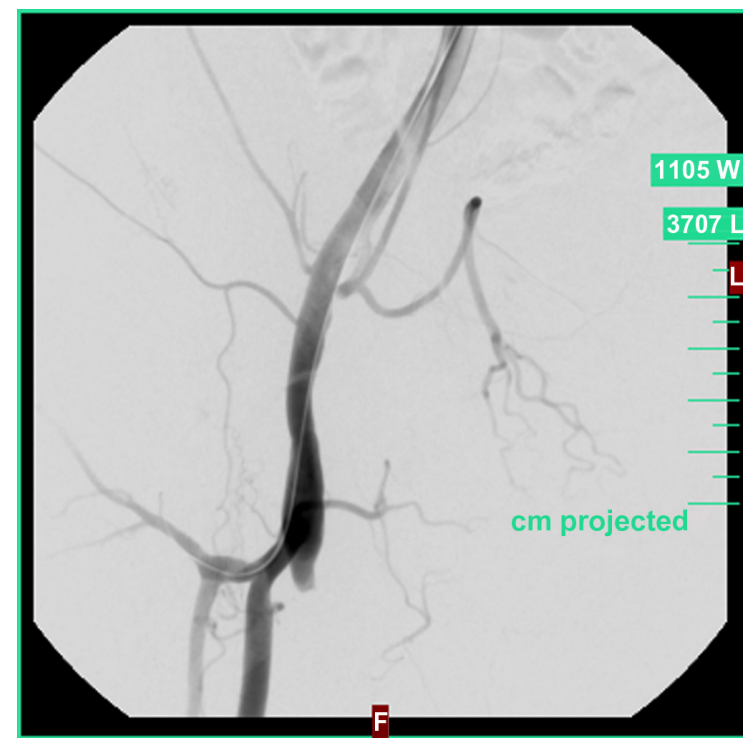

Figure 3 Acute proximal occlusion of a femoro-popliteal bypass on right. 


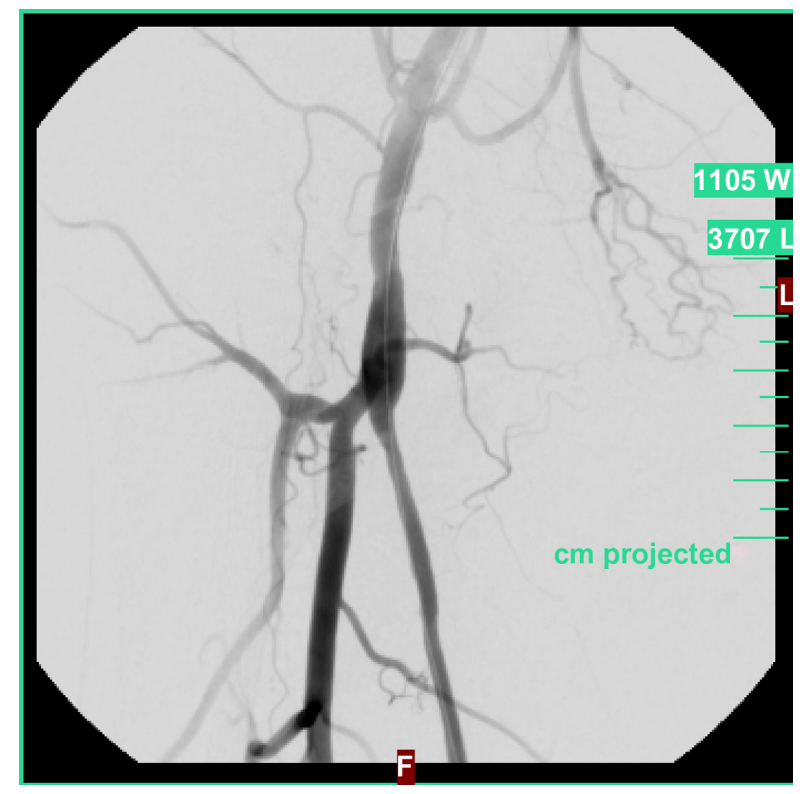

Figure 4 Reperfusion after two rotation thrombectomy passes.

Clinically, none of the patients could be classified in a Rutherford stage greater than II. The average ABI value was $0.81 \pm 0.1$. Average walking capacity was $323 \mathrm{~m}$. No re-interventions were necessary in any of the patients during this 6-month period (Table 3).

\section{Efficacy analysis of the Rotarex system in the indication of acutely occluded femoropopliteal bypasses}

To date, there has been no extensive, systematic scientific review of the Rotarex system's use in acutely occluded

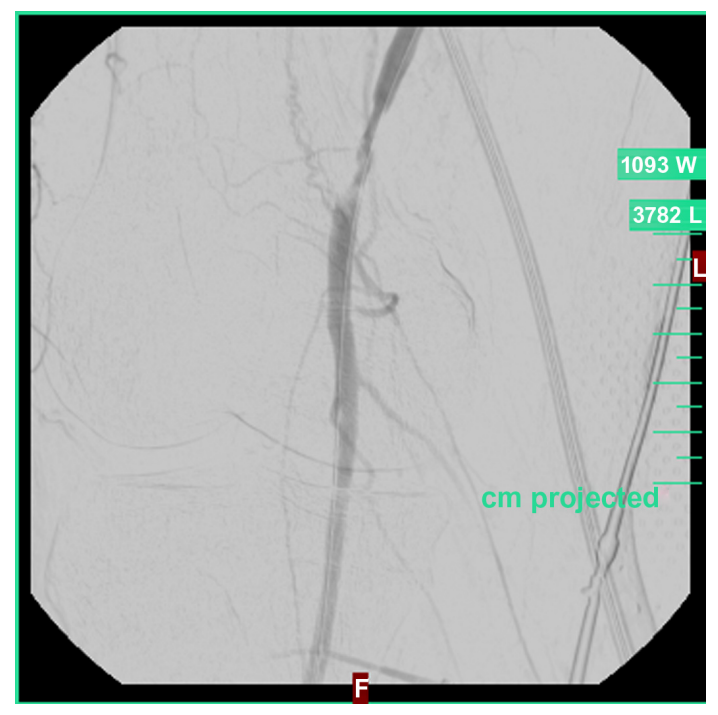

Figure 5 Detection of a high-grade insertion stenosis of the bypass as the presumed cause of the occlusion.

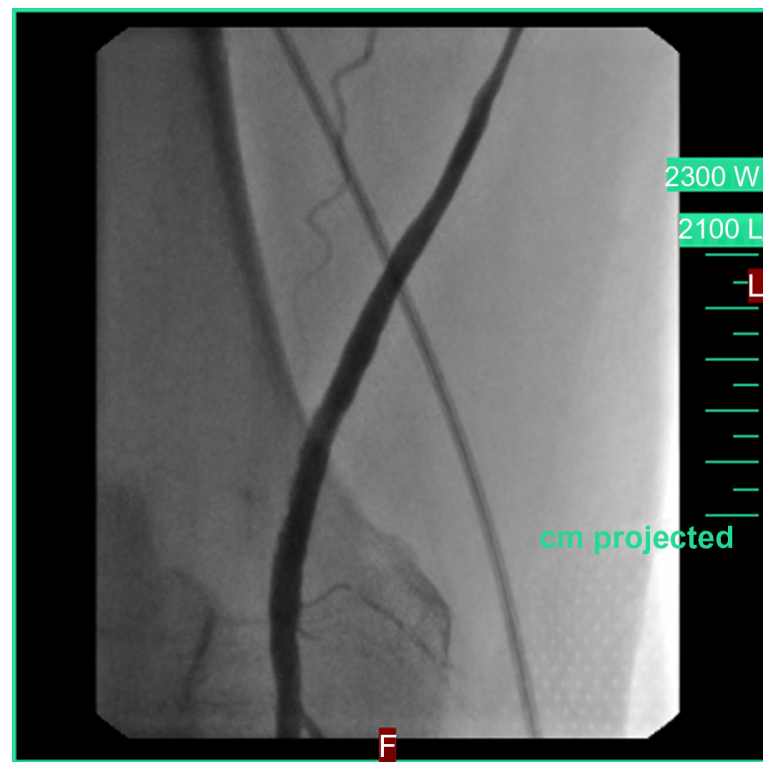

Figure 6 Normal flow in the bypass following stent percutaneous transluminal angioplasty.

femoropopliteal bypasses, since only monocentric studies and individual case studies have been reported. A paper by Wissgott et $\mathrm{al}^{10}$ demonstrated, in a direct comparison between the Rotarex system and the EKOS Lysus ${ }^{\circledR}$ peripheral catheter system, a technical success rate of $100 \%$ for the Rotarex system in this indication, and also documented a significantly shortened procedural time with the Rotarex system. Patients with femoropopliteal bypasses that had been occluded for up to 14 days were included in the particular treatment regimen. A majority of the patients were found to be in stage I or IIa of acute ischemia of the extremities. The 8 F Rotarex catheter was used in most cases (twelve patients), while the other occluded bypasses were successfully reopened with the $6 \mathrm{~F}$ Rotarex catheter. The Zeller Working Group likewise reports a high rate of technical success following rotation thrombectomy. ${ }^{7}$ By performing a stent-optimized angioplasty following the thrombectomy, a high percentage (78\%) of very good early functional results were achieved (see Table 4). We can report similarly positive results with a PMT-based technical success rate of $100 \%$ in the collective we observed. Except for four patients, local lysis was not necessary due to significant residual thrombi still in situ. In cases of inadequate removal of the thrombus load, local lysis was performed following the PMT with tissue plasminogen activator (Actilyse ${ }^{\circledR}$ ) using a pulse-spray catheter. Due to the PMT conducted beforehand, the thrombus load was usually so greatly reduced that only very low-dose lysis was necessary (t-PA bolus $5 \mathrm{mg}, 1-3 \mathrm{mg} / \mathrm{h}$ ). Through this form of hybrid therapy, good long-term courses were able to be documented, 

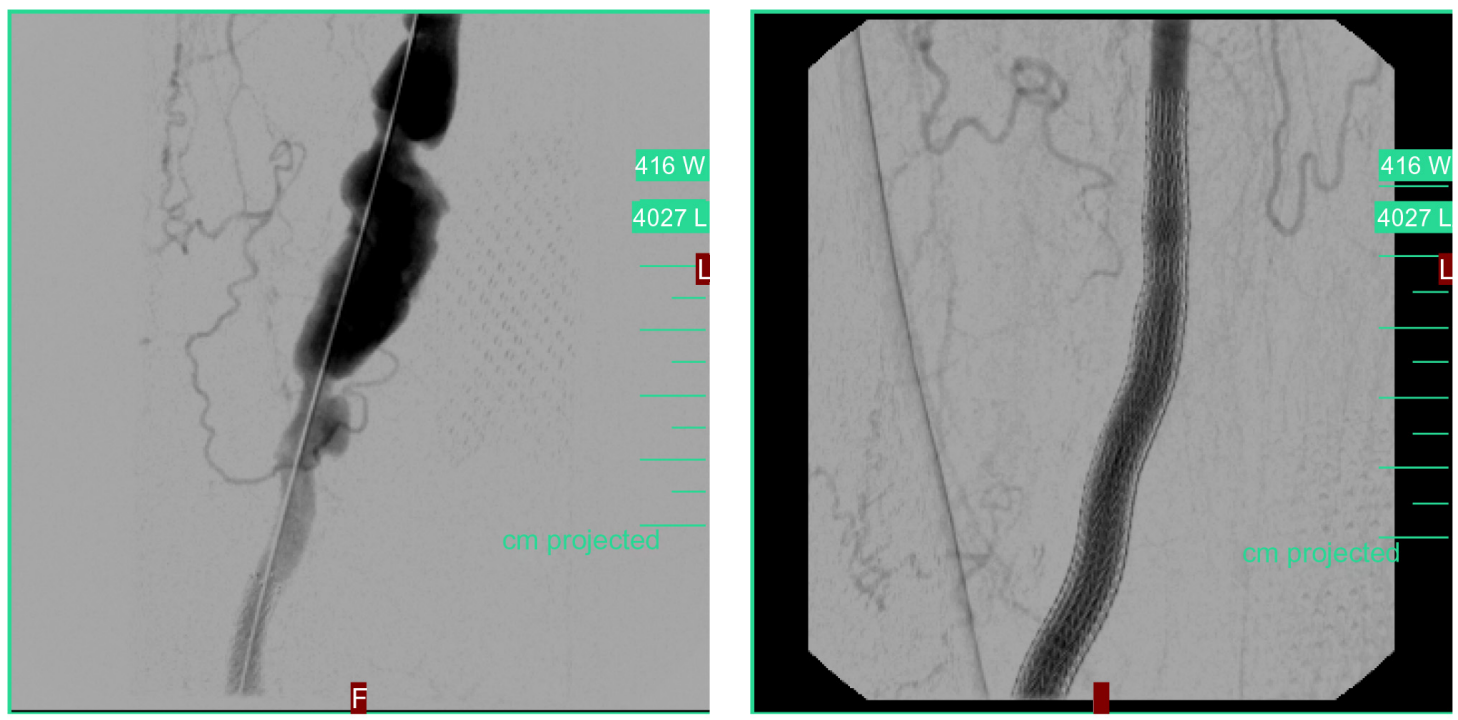

Figure 7 Degeneratively altered femoropopliteal venous bypass on right following acute reopening with rotation thrombectomy (Rotarex $8 \mathrm{~F}$ ). Notes: Large bypass aneurysm upstream of the distal anastomosis. Elimination of the aneurysm in two steps using a Viabahn ${ }^{\circledR}$ endoprosthesis.

even in larger native vessel areas (aorta, pelvic circulation). ${ }^{5}$ In several cases, a high-grade, hemodynamically relevant insertion stenosis was able to documented as the cause of the femoropopliteal bypass occlusion, which was then treated in the same session. In the follow-up observation of our collective, no renewed bypass occlusions or hemodynamically relevant restenoses occurred within a period of 6 months. After 6 months, the ABI value was $0.81 \pm 0.1$. The data correspond to the empirical values of Wissgott et al, ${ }^{6,10}$ who followed their patient collective over 12 months and describe a primary patency rate of $66 \%$ and a secondary patency rate of $86 \%$. Zeller et al assessed the course of their study collective significantly more critically because in these patients a restenosis rate of $86 \%$ at 12 months was determined. We can only speculate about the cause of this. A possible reason is surely the fact that, in the collective of Zeller et al, only patients with an artificial bypass were examined. ${ }^{7,8}$ In the collective we describe and in that of Wissgott et al, the percentage of artificial bypass thrombectomies was significantly smaller. The cause of the elevated reintervention rate for PTFE bypasses lies in the "foreign body reaction" of the vessels, which has been scientifically proven and recognized. ${ }^{11}$ Since the restenosis rate in the collective of

Table 3 Six-month follow-up data

\begin{tabular}{ll}
\hline Followed up patients & $2 \mathrm{I} / 22$ \\
Rutherford stage & I (I5 patients) \\
Walking capacity & II $(6$ patients $)$ \\
Ankle brachial index (ABI) & $323 \mathrm{~m}$ \\
Death & $0.8 \mathrm{I} \pm 0 . \mathrm{I}$ \\
Reinterventions & $0 / 22$ \\
\hline
\end{tabular}

Zeller et al suddenly jumped after 6 months, one of the causes may lie in a proliferative restenosis of the anastomosis area, making repeat interventions or operations necessary.

A great advantage of the PMT system compared with local lysis is the minimization of systemic bleeding complications, which were documented in particular in the large lysis studies. ${ }^{2,3}$

In our collective, there were no larger bleeding complications except for smaller inguinal hematomas. The patients we treated only had to stay in the intensive care unit for a few hours and were able to leave the hospital, on average, after 3.4 days. In some cases, in patients in whom a cardioembolic event was suspected, embolism source diagnostics were later conducted.

Complications, in general, using the rotational thrombectomy device are described. In some patients part of the thrombus could be advanced further down in the treated vessel, which makes a local lysis therapy necessary especially in the crural vessels. Puncture-associated complications increase with the $8 \mathrm{~F}$ Rotarex system, like groin hematoma, aneurysm, and bleeding complications. This is even more likely in patients who need additional local lysis therapy. In our opinion, the technical problems of the Rotarex system reported in earlier papers, ${ }^{7}$ particularly the danger of helix breakage in acute aortoiliac bifurcations, no longer exist in the current generation of the system. Especially through a further development of the enveloping shaft material with high flexibility, the danger of helix breakage in crossover use appears to be significantly reduced in the most up-to-date Rotarex version. Because of this, acute aortoiliac angles no 
Table 4 Follow-up findings after rotation thrombectomy

\begin{tabular}{|c|c|c|c|}
\hline Study & Number of patients & Technical success rate & Follow-up \\
\hline \multirow[t]{2}{*}{ Zeller et $\mathrm{al}^{7}$} & 7 & $78 \%(7 / 9)$ & $0.90 \pm 0.10(\mathrm{ABI}$ at 3 months $)$ \\
\hline & & & Restenosis at 12 months: $86 \%$ \\
\hline \multirow[t]{4}{*}{ Wissgott et al ${ }^{10}$} & 20 & $95 \%(19 / 20)$ & Primary patency rate: $66 \%$ \\
\hline & & & (12 \pm 3 months) \\
\hline & & & Secondary patency rate: $86 \%$ \\
\hline & & & (12 \pm 3 months) \\
\hline Wissgott et $\mathrm{a}^{6}$ & 10 & $100 \%(10 / 10)$ & $0.85 \pm 0.10(\mathrm{ABI}$ at I month $)$ \\
\hline \multirow[t]{2}{*}{ Lichtenberg et al 201 I } & 22 & $82 \%(18 / 22)$ & $0.8 \mathrm{I} \pm 0 . \mathrm{I}(\mathrm{ABI}$ at 6 months $)$ \\
\hline & & & No reinterventions after 6 months \\
\hline
\end{tabular}

Abbreviation: $\mathrm{ABI}$, ankle-brachial index.

longer pose a significant problem in comparison with the earlier Rotarex generation. ${ }^{5}$ The incidence of device-associated vessel perforations or dissections is low when care is taken with the intravascular course of the guide wire and it is monitored. In a collective of 40 patients from our clinic with thrombectomy, each in native vessels, uncomplicated dissection was shown in five of the patients. No perforation with a necessary (covered) stent implantation occurred. None of this type of complication occurred in the collective reported here. Experience has shown that there is a particularly greater risk in highly calcified vessels, the cause here appearing to be the aspiration of a hard calcified plaque that catches in the inlet of the helix. This can cause the rotating helix to produce a strong pull on the arterial wall, which can lead to perforation.

Rotation thrombectomy is included in the German DRG system. The procedure "rotation thrombectomy" is listed under DRG F 59 A for diagnosis I 74.3 (embolic occlusion).

\section{Summary}

The Rotarex system offered by Straub appears to be an effective alternative in the treatment of acute and subacute femoropopliteal bypass occlusions in comparison to established therapies such as vascular surgery and local lysis. The system is available for use to an experienced intervention team within a few minutes and is simple to handle. Because of the offer of both $6 \mathrm{~F}$ and $8 \mathrm{~F}$ devices, the appropriate system for the corresponding thrombus load can be used. Whenever possible, we recommend using the $8 \mathrm{~F}$ Rotarex system in the indication of femoropopliteal bypass occlusion in order to reduce the thrombus load as much as possible and to avoid any additional lysis. Therefore, an effective interventional treatment possibility is available to the affected patient, which avoids open vascular surgery. Especially in patients who are not candidates for an open procedure due to their comorbidities, this technique seems to be a promising interventional alternative.
The data should be considered as a presentation of preliminary data and results with the Rotarex device for femoropopliteal bypass acute occlusion in a small group of patients from a single center. As no control group was created, ie, a group of patients with occluded femoropopliteal bypasses treated with conventional (surgical) techniques, we were not able to compare both strategies directly. Our next step will be to plan a randomized, multicenter, prospective trial with direct comparison of interventional and surgical treatment to answer these questions.

\section{Consequences for clinic and practice}

Acute ischemia of the extremities represents a risk for the entire body in addition to the danger for the affected limb, since organs such as the kidneys, heart, and brain may also suffer due to the anaerobic metabolic situation caused by the hypoperfusion. Therefore, rapid diagnosis and an effective revascularization strategy are important measures for the patient. In these cases, percutaneous mechanical thrombectomy is an effective and safe endovascular therapy option in addition to the established lysis therapy.

\section{Disclosure}

The authors report no conflicts of interest in this work.

\section{References}

1. Ouriel K, Shortell CK, De Weese JA, et al. A comparison of thrombolytic therapy with operative revascularization in the initial treatment of acute peripheral arterial ischemia. J Vasc Surg. 1994;19:1021-1030.

2. The STILE Trial: results of a prospective randomized trial evaluating surgery versus thrombolysis for ischemia of the lower extremity. Ann Surg. 1994;220:251-266.

3. Ouriel K, Veith FJ, Sasahara AA; for the Thrombolysis or Peripheral Arterial Surgery (TOPAS) investigators. A comparison of recombinant urokinase with vascular surgery as initial treatment for acute arterial occlusion of the legs. N Engl J Med. 1998;338(16):1105-1111.

4. Norgen L, Hiatt WR, Dormandy JA, et al. Inter-Society Consensus for the Management of Peripheral Arterial Disease (TASC II). Eur J Vasc Endovasc Surg. 2007;33:S1-S75. 
5. Lichtenberg M. Percutaneous mechanical thrombectomy by means of rotational thrombectomy. Current Study situation. Med Klin (Munich). 2010;105:705-710.

6. Wissgott C, Kamusella P, Richter A, Klein-Weigel P, Steinkamp HJ. Mechanical rotational thrombectomy for treatment thrombolysis in acute and subacute occlusion of femoropopliteal arteries: retrospective analysis of the results from 1999 to 2005. Rofo. 2008;180:325-331.

7. Zeller T, Frank U, Bürgelin K, et al. Early experience with a rotationel thrombectomy device for treatment of acute and subacute infra-aortic arterial occlusions. $J$ Endovasc Ther. 2003;10:322-331.

8. Zeller T, Frank U, Bürgelin K, et al. Long-term results after recanalization of acute and subacute thrombotic occlusions of the infra-aortic arteries and bypass-grafts using a rotational thrombectomy device. Rofo. 2002;174:1559-1565.
9. Duc SR, Schoch E, Pfyffer M, et al. Recanalisation of acute and subacute femoropopliteal artery occlusions with the rotarex catheter: one year follow-up, single center experience. Cardiovasc Intervent Radiol. 2005;28:603-610.

10. Wissgott C, Kamusella P, Richter A, Klein-Weigel P, Schink T, Steinkamp HJ. Treatment of acute femoropopliteal bypass graft occlusion: comparison of mechanical rotational thrombectomy with ultrasound-enhanced lysis. Rofo. 2008;180:547-552.

11. Neumayer Ch, Panhofer P, Nanobashvili J, Polterauer P. Therapeutic options on femoral artery occlusion: indications, techniques and result - a vascular surgeon's view. Z Gefäßmed. 2005;2(3):4-11.
Vascular Health and Risk Management

\section{Publish your work in this journal}

Vascular Health and Risk Management is an international, peerreviewed journal of therapeutics and risk management, focusing on concise rapid reporting of clinical studies on the processes involved in the maintenance of vascular health; the monitoring, prevention and treatment of vascular disease and its sequelae; and the involvement of

\section{Dovepress}

metabolic disorders, particularly diabetes. This journal is indexed on PubMed Central and MedLine. The manuscript management system is completely online and includes a very quick and fair peer-review system, which is all easy to use. Visit http://www.dovepress.com/ testimonials.php to read real quotes from published authors.

Submit your manuscript here: http://www.dovepress.com/vascular-health-and-risk-management-journal 